\title{
La entrevista Cannabis Abuse Screening Test (CAST) para el diagnóstico de trastornos psiquiátricos en jóvenes consumidores de cannabis
}

\section{The Cannabis Abuse Screening Test (CAST) for psychiatric disorders diagnosis in young cannabis users}

\author{
Aida M. Cuenca-Royo*,**; Albert Sánchez- \\ NIUBÓ ${ }^{\star, * * ;}$ MARTA TORRENS ${ }^{\star \star *, * \star * *}$; JOSEP M. \\ SUELVES ${ }^{\star \star \star \star *}$; ANTÒNIA DOMINGO-SALVANY ${ }^{\star, \star *}$
}

* Drug Abuse Epidemiology Research Group, IMIM-Hospital del Mar.

${ }^{*}$ CIBER en Epidemiología y Salud Publica (CIBERESP

*** Institut de Neuropsiquiatria i Addiccions, Hospital del Mar, Barcelona.

**** Department of Psychiatry, Universitat Autònoma de Barcelona.

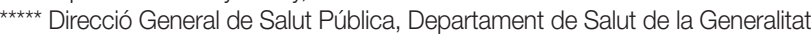
de Catalunya.

Enviar correspondencia a:

Antònia Domingo-Salvany

IMIM-Hospital del Mar. Dr Aiguader 88

08003 Barcelona. España.

Teléfono: 3493 3160700;

Fax: 34933160797.

Email: adomingo@imim.es
$\mathrm{E}$ cannabis es la droga de uso ilegal más consumida en España, especialmente entre los jóvenes (15-34 años) con una prevalencia del 19.4\% (European Monitoring Centre for Drugs and Drug Addiction, 2011).

Diferentes estudios sobre consumo de sustancias y patología psiquiátrica han mostrado que existe una relación entre los trastornos por uso de sustancias (TUS) y los trastornos del eje I y II (no-TUS), tales como los del estado del ánimo, de ansiedad, límite o antisocial de la personalidad, etc. (Wittchen et al., 2007). Dado el elevado consumo de cannabis y su relación con la presencia de patología psiquiátrica, es necesario encontrar estrategias para detectar la presencia de algún trastorno psiquiátrico en estos consumidores.

La Cannabis Abuse Screening Test (CAST) (Legleye, Karila, Beck y Reynaud, 2007) es una escala de cribado de consumo problemático de cannabis, corta y fácil de administrar, recientemente validada en castellano. Ha demostrado tener buenas propiedades psicométricas para evaluar la severidad de dependencia de cannabis teniendo en cuenta diferentes componentes de validez en jóvenes y en jóvenes-adultos (Fernández-Artamendi, Fernández-Hermida, Muniz-Fernandez, Secades-Villa y García-Fernández, 2012; Cuenca-Royo et al.,
2012). Está formada por 6 ítems y evalúa la frecuencia de los siguientes eventos en los últimos 12 meses: uso recreativo (1."¿Has fumado cannabis antes del mediodía?"; 2.-"¿Has fumado cannabis cuando estabas solo?"), problemas de memoria (3."¿Has tenido problemas de memoria al fumar cannabis?"), si le animan a reducir o dejar de consumir cannabis (4.-"¿Te han dicho los amigos o miembros de tu familia que deberías reducir el consumo de cannabis?"), los intentos fallidos para dejarlo (5.-"¿Has tratado de reducir o dejar de consumir cannabis sin conseguirlo?"), y los problemas relacionados con el consumo de cannabis (6.- "¿Has tenido problemas debido a tu consumo de cannabis (disputa, pelea, accidente, mal resultado escolar, etc. )?"). Cada ítem se responde en una escala tipo Likert (0 "nunca", 1 "raramente", 2 "algunas veces", 3 "bastante a menudo" y 4 "muy a menudo"). En su versión completa (CAST-f), la puntuación es la suma de la puntuación en cada ítem, proporcionando un rango de 0 a 24. En el estudio de Cuenca-Royo se observó que esta versión completa ofrece más información y presenta mejor validez de criterio que la versión binaria propuesta por los autores originales(Cuenca-Royo et al., 2012).

Objetivo: Comparar las medias de CAST-f de sujetos sin diagnóstico psiquiátrico, con sólo TUS, sólo trastornos del eje I y/o II o con ambas (TUS+no-TUS). 
Participantes: 241 voluntarios (18-25 años), con un amplio espectro de consumo de cannabis en los últimos 12 meses, sin TUS para otras drogas ilegales.

Método: Autoadministración de la CAST y administración de la versión española de la "Psychiatric Interview for Substance and Mental Disorders" (PRISM) (Torrens, Serrano, Astals, Perez-Dominguez y Martin-Santos, 2004) entrevista semiestructurada que evalúa, según criterios DSM-IV, además de los TUS, los siguientes trastornos del Eje I: del estado de ánimo, de ansiedad, psicóticos, de la conducta alimentaria, inducidos por sustancias y por déficit de atención con hiperactividad; y 2 trastornos del Eje II: antisocial y límite de la personalidad. Al resto de trastornos del eje I y a los del eje II los denominamos trastornos no-TUS.

La próxima versión del Manual Diagnóstico y Estadístico de los Trastornos Mentales (DSM-V) (American Psychiatric Association, 2011) no contempla la distinción entre abuso y dependencia de sustancias y recomiendan su combinación en un solo trastorno de gravedad clínica graduada agrupando todos los criterios de dependencia y todos los de abuso, con excepción al legal. En el caso del cannabis, se añadirá también el criterio de abstinencia, algo que no se consideraba para esta sustancia en la versión IV. Para recibir el diagnóstico de adicción y trastornos relacionados una persona deberá cumplir al menos 2 criterios (2-3 criterios: adicción moderada, 4 o más: adicción severa).

Los puntos de corte para CAST referidos en el estudio de Cuenca-Royo et al. fueron 9 para dependencia (DSM-IV) y 7 para adicción moderada (proxi DSM-V).

Más detalles de la muestra y la metodología pueden consultarse en el artículo original (Cuenca-Royo et al., 2012).

Resultados: Las medias de la CAST-f fueron para sólo no-TUS $5.56($ IC95\%=2.89-8.22; $n=9)$; para sólo TUS 10.88 $($ IC $95 \%=10.12-11.63 ; n=105) ;$ para TUS/No-TUS 12.58 (IC95\%=10.44-14.72; $n=12)$. Aquellos sujetos que no tenían ningún trastorno $(n=115)$ tuvieron una media de 6.77 (IC95\%=6.04-7.49). La figura 1 muestra que aquellos jóvenes adultos con puntuaciones más altas en la CAST-f presentaban algún trastorno psiquiátrico según la PRISM. Los jóvenes que presentaron TUS de cannabis se distribuyeron mayoritariamente en los rangos más altos. La prevalencia en el punto de corte, según criterios DSM-IV (9) para TUS de cannabis fue del $68 \%$, y para adicción moderada con un proxi del DSM-V (7) del $63 \%$.

Discusión: La hipótesis inicial era que a mayor puntuación de la CAST aparecería mayor prevalencia tanto de trastornos psiquiátricos TUS como de no-TUS, pero los consumidores de cannabis que sólo presentaban trastornos no-TUS tuvieron puntuaciones menores.

Conclusiones: La CAST es una escala sencilla y de fácil administración, su utilización en atención primaria permitiria detectar los jóvenes que podrían estar en riesgo de presentar trastorno por uso de cannabis. Además, puede ayudar a orientar el diagnóstico y dirigir a programas de tratamiento específicos.

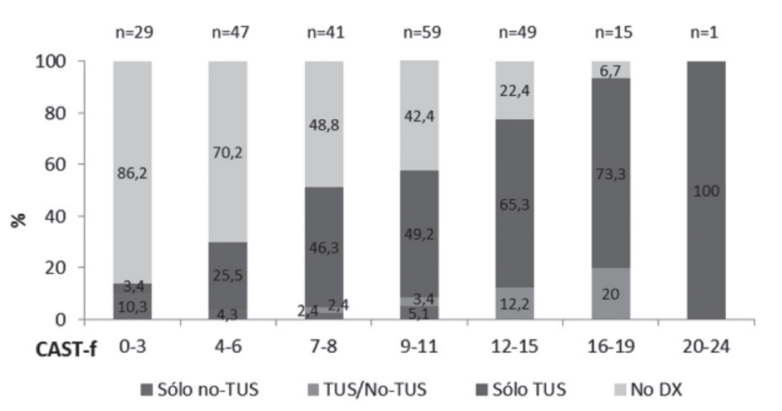

Figura 1. Distribución de trastornos psiquiátricos según las puntuaciones de la CAST.

\section{Agradecimientos}

Agradecemos la financiación de ETS (PI06/90491), FIS (PI070960), FIS-Redes de Investigación cooperativa RD12/0028/0009 y 0018 y AGAUR 2009 SGR 718. Albert Sánchez-Niubó está subvencionado por ISCIII CA08/00214.

\section{Referencias}

American Psychiatric Association. (2011). DSM-V Development.

Cuenca-Royo, A. M., Sanchez-Niubo, A., Forero, C. G., Torrens, M., Suelves, J. M. y Domingo-Salvany, A. (2012). Psychometric properties of the CAST and SDS scales in young adult cannabis users. Addictive Behaviors, 37, 709-715. doi:10.1016/j.addbeh.2012.02.012. Epub 2012 Feb 17.

European Monitoring Centre for Drugs and Drug Addiction (EMCDDA) (2011). 2011 Annual Report. The State of the Drugs Problem in Europe. Lisbon: Publications Office of the European Union, 2011. doi:10.2810/4450

Fernández-Artamendi, S., Fernández-Hermida, J. R., Muniz-Fernandez, J., Secades-Villa, R. y Garcia-Fernández, G. (2012). Screening of cannabis-related problems among youth: the CPO-A-S and CAST questionnaires. Substance Abuse Treatment, Prevention, and Policy 7, 13.doi: 10.1186/1747-597X-7-13.

Legleye, S., Karila, L., Beck, F. y Reynaud, M. (2007). Validation of the CAST, a general population Cannabis Abuse Screening Test. Journal of Substance Use 12, 233-242. doi: 10.1080/14659890701476532

Torrens, M., Serrano, D., Astals, M., Perez-Dominguez, G. y Martin-Santos, R. (2004). Diagnosing comorbid psychiatric disorders in substance abusers: validity of the Spanish versions of the Psychiatric Research Interview for Substance and Mental Disorders and the Structured Clinical Interview for DSM-IV. American Journal of Psychiatry, 161, 1231-1237. doi: 10.1176/appi.ajp.161.7.1231

Wittchen, H. U., Frohlich, C., Behrendt, S., Gunther, A., Rehm, J., Zimmermann, P. et al. (2007). Cannabis use and cannabis use disorders and their relationship to mental disorders: a 10-year prospective-longitudinal community study in adolescents. Drug and Alcohol Dependence, 88 Suppl 1, S60-S70. doi: 10.1016/j. drugalcdep.2006.12.013 